\title{
Trim-Commanded Adaptive Control for Waypoint-Defined Trajectory Following
}

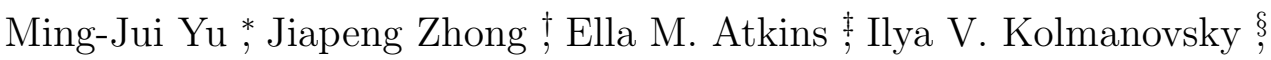 \\ and Dennis S. Bernstein \\ University of Michigan, 1320 Beal Ave., Ann Arbor, MI 48109
}

\begin{abstract}
In the event of unforeseen aircraft faults, safe and timely recovery may depend on multiple layers of decision-making. This paper applies retrospective cost adaptive control (RCAC) to the inner control loop of an aircraft's safety-management system and investigates the ability to follow sequences of trim commands given by higher level flight planners. We first show that trim conditions can be realized by commanding constant airspeed, flight path angle, and turn rate, and then investigate the ability of RCAC to follow possibly conflicting trim commands with minimal modeling of the static flight envelope.
\end{abstract}

\section{Introduction}

The shortest planar path between two points subject to curvature constraints is given by a Dubins path [1]. These solutions, which consist of straight line segments and circular arcs, have been extended and applied to both ground and air vehicles with realistic vehicle dynamics [2-4]. For aircraft flight, straight line segments and circular arcs between waypoints can be realized as trim flight conditions, which can be specified in terms of air speed, flight path angle, and turn radius. The dynamics of a realistic aircraft must be accounted for in the transitions at waypoints [5].

In the present paper we use Dubins paths with waypoints for emergency flight control. The goal is to follow a sequence of trim commands with minimal modeling information to account for possible failures and uncertainties in the aircraft dynamics and static flight envelope. To do this, we apply retrospective cost adaptive control (RCAC) with airspeed, flight path angle, and turn radius commands switched by ramp transitions at waypoints. The trim commands are not based on knowledge of the static flight envelope, which may be uncertain. Consequently, the trim commands are potentially conflicting.

RCAC has been developed in [6-10] and applied to aircraft flight control in [11]. As in [11], we use the nonlinear NASA Generic Transport Model (GTM) $[12,13]$ to investigate the ability of RCAC to follow a sequence of trim commands. To transition between waypoints, we use ramp commands to mitigate the path-following error due to the aircraft dynamics. Of particular interest is the evolution of the controller gains as RCAC adapts to changing trim commands under envelope uncertainty.

\footnotetext{
*Graduate Student, Aerospace Engineering Department, University of Michigan

${ }^{\dagger}$ Graduate Student, Control Science and Engineering Department, Harbin Institute of Technology, Harbin, China

¥Associate Professor, Aerospace Engineering Department, University of Michigan

$\S$ Professor, Aerospace Engineering Department, University of Michigan

ฯProfessor, Aerospace Engineering Department, University of Michigan
} 


\section{Adaptive Controller Formulation}

\section{II.A. Problem Formulation}

Consider the MIMO discrete-time system

$$
\begin{aligned}
x(k+1) & =A x(k)+B u(k)+D_{1} w(k), \\
y(k) & =C x(k)+D_{2} w(k), \\
z(k) & =E_{1} x(k)+E_{0} w(k),
\end{aligned}
$$

where $x(k) \in \mathbb{R}^{n}, y(k) \in \mathbb{R}^{l_{y}}, z(k) \in \mathbb{R}^{l_{z}}, u(k) \in \mathbb{R}^{l_{u}}, w(k) \in \mathbb{R}^{l_{w}}$, and $k \geq 0$. Our goal is to develop an adaptive output feedback controller that minimizes the performance variable $z$ in the presence of the exogenous signal $w$ with minimal modeling information about the dynamics and $w$. Note that $w$ can represent either a command signal to be followed, an external disturbance to be rejected, or both. The system (1)-(3) can represent a sampled-data application arising from a continuous-time system with sample and hold operations.

If $D_{1}=0$ and $E_{0} \neq 0$, then the objective is to have the output $E_{1} x$ follow the command signal $-E_{0} w$. On the other hand, if $D_{1} \neq 0$ and $E_{0}=0$, then the objective is to reject the disturbance $w$ from the performance measurement $E_{1} x$. Furthermore, if $D_{1}=\left[\begin{array}{cc}\hat{D}_{1} & 0\end{array}\right], E_{0}=\left[\begin{array}{ll}0 & \hat{E}_{0}\end{array}\right]$, and $w(k)=$ $\left[w_{1}(k)^{\mathrm{T}} w_{2}(k)^{\mathrm{T}}\right]^{\mathrm{T}}$, then the objective is to have $E_{1} x$ follow the command $-\hat{E}_{0} w_{2}$ while rejecting the disturbance $w_{1}$. Lastly, if $D_{1}$ and $E_{0}$ are empty matrices, then the objective is output stabilization, that is, convergence of $z$ to zero.

\section{II.B. Retrospective Cost}

For $i \geq 1$, define the Markov parameter of $G_{z u}$ given by

$$
H_{i} \triangleq E_{1} A^{i-1} B
$$

For example, $H_{1}=E_{1} B$ and $H_{2}=E_{1} A B$. Let $r$ be a positive integer. Then, for all $k \geq r$,

$$
x(k)=A^{r} x(k-r)+\sum_{i=1}^{r} A^{i-1} B u(k-i)+\sum_{i=1}^{r} A^{i-1} D_{1} w(k-i),
$$

and thus

$$
z(k)=E_{1} A^{r} x(k-r)+\sum_{i=1}^{r} E_{1} A^{i-1} D_{1} w(k-i)+E_{0} w(k)+\bar{H} \bar{U}(k-1),
$$

where

$$
\bar{H} \triangleq\left[\begin{array}{lll}
H_{1} & \cdots & H_{r}
\end{array}\right] \in \mathbb{R}^{l_{z} \times r l_{u}}
$$

and

$$
\bar{U}(k-1) \triangleq\left[\begin{array}{c}
u(k-1) \\
\vdots \\
u(k-r)
\end{array}\right]
$$


Next, we rearrange the columns of $\bar{H}$ and the components of $\bar{U}(k-1)$ and partition the resulting matrix and vector so that

$$
\bar{H} \bar{U}(k-1)=\mathcal{H}^{\prime} U^{\prime}(k-1)+\mathcal{H} U(k-1),
$$

where $\mathcal{H}^{\prime} \in \mathbb{R}^{l_{z} \times\left(r l_{u}-l_{U}\right)}, \mathcal{H} \in \mathbb{R}^{l_{z} \times l_{U}}, U^{\prime}(k-1) \in \mathbb{R}^{r l_{u}-l_{U}}$, and $U(k-1) \in \mathbb{R}^{l_{U}}$. Then, we can rewrite (6) as

$$
z(k)=\mathcal{S}(k)+\mathcal{H} U(k-1),
$$

where

$$
\mathcal{S}(k) \triangleq E_{1} A^{r} x(k-r)+\sum_{i=1}^{r} E_{1} A^{i-1} D_{1} w(k-i)+E_{0} w(k)+\mathcal{H}^{\prime} U^{\prime}(k-1) .
$$
the form

Next, for $j=1, \ldots, s$, we rewrite (8) with a delay of $k_{j}$ time steps, where $0 \leq k_{1} \leq k_{2} \leq \cdots \leq k_{s}$, in

$$
z\left(k-k_{j}\right)=\mathcal{S}_{j}\left(k-k_{j}\right)+\mathcal{H}_{j} U_{j}\left(k-k_{j}-1\right)
$$

where (9) becomes

$$
\mathcal{S}_{j}\left(k-k_{j}\right) \triangleq E_{1} A^{r} x\left(k-k_{j}-r\right)+\sum_{i=1}^{r} E_{1} A^{i-1} D_{1} w\left(k-k_{j}-i\right)+E_{0} w\left(k-k_{j}\right)+\mathcal{H}_{j}^{\prime} U_{j}^{\prime}\left(k-k_{j}-1\right),
$$

and (7) becomes

$$
\bar{H} \bar{U}\left(k-k_{j}-1\right)=\mathcal{H}_{j}^{\prime} U_{j}^{\prime}\left(k-k_{j}-1\right)+\mathcal{H}_{j} U_{j}\left(k-k_{j}-1\right),
$$

where $\mathcal{H}_{j}^{\prime} \in \mathbb{R}^{l_{z} \times\left(r l_{u}-l_{U_{j}}\right)}, \mathcal{H}_{j} \in \mathbb{R}^{l_{z} \times l_{U_{j}}}, U_{j}^{\prime}\left(k-k_{j}-1\right) \in \mathbb{R}^{r l_{u}-l_{U_{j}}}$, and $U_{j}\left(k-k_{j}-1\right) \in \mathbb{R}^{l_{U_{j}}}$. Now, by stacking $z\left(k-k_{1}\right), \ldots, z\left(k-k_{s}\right)$, we define the extended performance

$$
Z(k) \triangleq\left[\begin{array}{c}
z\left(k-k_{1}\right) \\
\vdots \\
z\left(k-k_{s}\right)
\end{array}\right] \in \mathbb{R}^{s l_{z}} .
$$

Therefore,

$$
Z(k) \triangleq \tilde{\mathcal{S}}(k)+\tilde{\mathcal{H}} \tilde{U}(k-1)
$$

where

$$
\tilde{\mathcal{S}}(k) \triangleq\left[\begin{array}{c}
\mathcal{S}_{1}\left(k-k_{1}\right) \\
\vdots \\
\mathcal{S}_{s}\left(k-k_{s}\right)
\end{array}\right] \in \mathbb{R}^{s l_{z}},
$$

$\tilde{U}(k-1)$ has the form

$$
\tilde{U}(k-1) \triangleq\left[\begin{array}{c}
u\left(k-q_{1}\right) \\
\vdots \\
u\left(k-q_{l_{\tilde{U}}}\right)
\end{array}\right] \in \mathbb{R}^{l_{\tilde{U}}},
$$

where, for $i=1, \ldots, l_{\tilde{U}}, k_{1} \leq q_{i} \leq k_{s}+r$, and $\tilde{\mathcal{H}} \in \mathbb{R}^{s l_{z} \times l_{\tilde{U}}}$ is constructed according to the structure of $\tilde{U}(k-1)$. The vector $\tilde{U}(k-1)$ is formed by stacking $U_{1}\left(k-k_{1}-1\right), \ldots, U_{s}\left(k-k_{s}-1\right)$ and removing copies of repeated components. 
Next, we define the retrospective performance

$$
\hat{z}\left(k-k_{j}\right) \triangleq \mathcal{S}_{j}\left(k-k_{j}\right)+\mathcal{H}_{j} \hat{U}_{j}\left(k-k_{j}-1\right),
$$

where the past controls $U_{j}\left(k-k_{j}-1\right)$ in (10) are replaced by the surrogate controls $\hat{U}_{j}\left(k-k_{j}-1\right)$. In analogy with (13), the extended retrospective performance for (17) is defined as

$$
\hat{Z}(k) \triangleq\left[\begin{array}{c}
\hat{z}\left(k-k_{1}\right) \\
\vdots \\
\hat{z}\left(k-k_{s}\right)
\end{array}\right] \in \mathbb{R}^{s l_{z}}
$$

and thus is given by

$$
\hat{Z}(k)=\tilde{\mathcal{S}}(k)+\tilde{\mathcal{H}} \hat{\tilde{U}}(k-1),
$$

where the components of $\hat{\tilde{U}}(k-1) \in \mathbb{R}^{l} \tilde{U}$ are the components of $\hat{U}_{1}\left(k-k_{1}-1\right), \ldots, \hat{U}_{s}\left(k-k_{s}-1\right)$ ordered in the same way as the components of $\tilde{U}(k-1)$. Subtracting (14) from (19) yields

$$
\hat{Z}(k)=Z(k)-\tilde{\mathcal{H}} \tilde{U}(k-1)+\tilde{\mathcal{H}} \hat{\tilde{U}}(k-1) .
$$

Finally, we define the retrospective cost function

$$
J(\hat{\tilde{U}}(k-1), k) \triangleq \hat{Z}^{\mathrm{T}}(k) R(k) \hat{Z}(k),
$$

where $R(k) \in \mathbb{R}^{l_{z} s \times l_{z} s}$ is a positive-definite performance weighting. The goal is to determine refined controls $\hat{\tilde{U}}(k-1)$ that would have provided better performance than the controls $U(k)$ that were applied to the system. The refined control values $\hat{\tilde{U}}(k-1)$ are subsequently used to update the controller.

\section{II.C. Cost Function Optimization with Adaptive Regularization}

To ensure that (21) has a global minimizer, we consider the regularized cost

$$
\bar{J}(\hat{\tilde{U}}(k-1), k) \triangleq \hat{Z}^{\mathrm{T}}(k) R(k) \hat{Z}(k)+\eta(k) R_{2} \hat{\tilde{U}}^{\mathrm{T}}(k-1) \hat{\tilde{U}}(k-1),
$$

where $\eta(k) \geq 0$, and $R_{2} \in \mathbb{R}^{\hat{U}} \geq 0$. Substituting (20) into (22) yields

$$
\bar{J}(\hat{\tilde{U}}(k-1), k)=\hat{\tilde{U}}(k-1)^{\mathrm{T}} \mathcal{A}(k) \hat{\tilde{U}}(k-1)+\hat{\tilde{U}}^{\mathrm{T}}(k-1) \mathcal{B}^{\mathrm{T}}(k)+\mathcal{C}(k),
$$

where

$$
\begin{aligned}
& \mathcal{A}(k) \triangleq \tilde{\mathcal{H}}^{\mathrm{T}} R(k) \tilde{\mathcal{H}}+\eta(k) R_{2} I_{l}, \\
& \mathcal{B}(k) \triangleq 2 \tilde{\mathcal{H}}^{\mathrm{T}} R(k)[Z(k)-\tilde{\mathcal{H}} \tilde{U}(k-1)], \\
& \mathcal{C}(k) \triangleq Z^{\mathrm{T}}(k) R(k) Z(k)-2 Z^{\mathrm{T}}(k) R(k) \tilde{\mathcal{H}} \tilde{U}(k-1)+\tilde{U}^{\mathrm{T}}(k-1) \tilde{\mathcal{H}}^{\mathrm{T}} R(k) \tilde{\mathcal{H}} \tilde{U}(k-1) .
\end{aligned}
$$

If either $\tilde{\mathcal{H}}$ has full column rank or $\eta(k)>0$ and $R_{2}>0$, then $\mathcal{A}(k)$ is positive definite. In this case, $\bar{J}(\hat{\tilde{U}}(k-1), k)$ has the unique global minimizer

$$
\hat{\tilde{U}}(k-1)=-\frac{1}{2} \mathcal{A}^{-1}(k) \mathcal{B}(k) .
$$




\section{II.D. Controller Construction}

The control $u(k)$ is given by the strictly proper time-series controller of order $n_{\mathrm{c}}$ given by

$$
u(k)=\sum_{i=1}^{n_{\mathrm{c}}} M_{i}(k) u(k-i)+\sum_{i=1}^{n_{\mathrm{c}}} N_{i}(k) y(k-i),
$$

where, for all $i=1, \ldots, n_{\mathrm{c}}, M_{i}(k) \in \mathbb{R}^{l_{u} \times l_{u}}$ and $N_{i}(k) \in \mathbb{R}^{l_{u} \times l_{y}}$. The control (28) can be expressed as

$$
u(k)=\theta(k) \phi(k-1),
$$

where

$$
\theta(k) \triangleq\left[M_{1}(k) \cdots M_{n_{\mathrm{c}}}(k) N_{1}(k) \cdots N_{n_{\mathrm{c}}}(k)\right] \in \mathbb{R}^{l_{u} \times n_{\mathrm{c}}\left(l_{u}+l_{z}\right)}
$$

and

$$
\phi(k-1) \triangleq\left[\begin{array}{c}
u(k-1) \\
\vdots \\
u\left(k-n_{\mathrm{c}}\right) \\
y(k-1) \\
\vdots \\
y\left(k-n_{\mathrm{c}}\right)
\end{array}\right] \in \mathbb{R}^{n_{\mathrm{c}}\left(l_{u}+l_{y}\right)}
$$

\section{II.E. Recursive Least Squares Update of $\theta(k)$} function

Next, let $d$ be a positive integer such that $\tilde{U}(k-1)$ contains $u(k-d)$ and define the cumulative cost

$$
J_{\mathrm{R}}(\theta, k) \triangleq \sum_{i=d+1}^{k} \lambda^{k-i}\left\|\phi^{\mathrm{T}}(i-d-1) \theta^{\mathrm{T}}(k)-\hat{u}^{\mathrm{T}}(i-d)\right\|^{2}+\lambda^{k}\left(\theta(k)-\theta_{0}\right) P_{0}^{-1}\left(\theta(k)-\theta_{0}\right)^{\mathrm{T}},
$$

where $\|\cdot\|$ is the Euclidean norm, and $\lambda \in(0,1]$ is the forgetting factor. Minimizing (32) yields

$$
\begin{array}{r}
\theta^{\mathrm{T}}(k)=\theta^{\mathrm{T}}(k-1)+\beta(k) P(k-1) \phi(k-d-1) \cdot\left[\phi^{\mathrm{T}}(k-d) P(k-1) \phi(k-d-1)+\lambda(k)\right]^{-1} \\
\cdot\left[\phi^{\mathrm{T}}(k-d-1) \theta^{\mathrm{T}}(k-1)-\hat{u}^{\mathrm{T}}(k-d)\right],
\end{array}
$$

where $\beta(k)$ is either zero or one. The error covariance is updated by

$$
\begin{array}{r}
P(k)=\beta(k) \lambda^{-1} P(k-1)+[1-\beta(k)] P(k-1)-\beta(k) \lambda^{-1} P(k-1) \phi(k-d-1) \\
\cdot\left[\phi^{\mathrm{T}}(k-d-1) P(k-1) \phi(k-d)+\lambda\right]^{-1} \phi^{\mathrm{T}}(k-d-1) P(k-1) .
\end{array}
$$

We initialize the error covariance matrix as $P(0)=\alpha I_{3 n_{\mathrm{c}}}$, where $\alpha>0$. Note that when $\beta(k)=0, \theta(k)=$ $\theta(k-1)$ and $P(k)=P(k-1)$. Therefore, setting $\beta(k)=0$ switches off the controller adaptation, and thus freezes the control gains. When $\beta(k)=1$, the controller is allowed to adapt.

\section{Defining Trim Conditions}

In this section, we define aircraft kinematic quantities in order to specify trim conditions. 


\section{III.A. Steady Flight}

The Earth inertial frame and the aircraft body-fixed frame are denoted by $\mathrm{F}_{\mathrm{E}}$ and $\mathrm{F}_{\mathrm{AC}}$, respectively. $\mathrm{F}_{\mathrm{AC}}$ is defined with $\hat{\imath}_{\mathrm{AC}}$ pointing out the nose, $\hat{\jmath}_{\mathrm{AC}}$ pointing out the right wing, and $\hat{k}_{\mathrm{AC}}$ is obtained from the cross product of $\hat{\imath}_{\mathrm{AC}}$ and $\hat{\jmath}_{\mathrm{AC}}$. These frames are related by a 3-2-1 Euler rotation sequence, which yields two intermediate frames $\mathrm{F}_{\mathrm{E}^{\prime}}$ and $\mathrm{F}_{\mathrm{E}^{\prime \prime}}$. The frames $\mathrm{F}_{\mathrm{AC}}$ and $\mathrm{F}_{\mathrm{E}}$ are therefore related by

$$
\mathrm{F}_{\mathrm{AC}}=\vec{R}_{\mathrm{AC} / \mathrm{E}} \mathrm{F}_{\mathrm{E}}=\vec{R}_{\hat{\mathrm{E}}_{\mathrm{E}^{\prime \prime}}}(\Phi) \vec{R}_{\hat{\jmath}_{\mathrm{E}^{\prime}}}(\Theta) \vec{R}_{\hat{k}_{\mathrm{E}}}(\Psi) \mathrm{F}_{\mathrm{E}}
$$

where $\vec{R}_{\hat{k}_{\mathrm{E}}}(\Psi)$ denotes an Euler rotation matrix about the $\hat{k}$ axis of $\mathrm{F}_{\mathrm{E}}, \vec{R}_{\hat{J}_{\mathrm{E}^{\prime}}}(\Theta)$ denotes an Euler rotation matrix about the $\hat{\jmath}$ axis of the frame $\mathrm{F}_{\mathrm{E}^{\prime}} \triangleq \vec{R}_{\hat{k}_{\mathrm{E}}}(\Psi) \mathrm{F}_{\mathrm{E}}$, and $\vec{R}_{\hat{\imath}_{\mathrm{E}^{\prime \prime}}}(\Phi)$ denotes an Euler rotation matrix about the $\hat{\imath}$ axis of the frame $\mathrm{F}_{\mathrm{E}^{\prime \prime}} \triangleq \vec{R}_{\hat{\jmath}_{\mathrm{E}^{\prime}}}(\Theta) \mathrm{F}_{\mathrm{E}^{\prime}}$.

The location of the center of mass of the aircraft relative to the center of the Earth $\mathrm{O}_{\mathrm{E}}$ is denoted by $\vec{r}_{\mathrm{c} / \mathrm{O}_{\mathrm{E}}}$. The velocity of the aircraft is therefore defined as

$$
\vec{V}_{\mathrm{AC}} \triangleq \vec{v}_{\mathrm{c} / \mathrm{O}_{\mathrm{E}} / \mathrm{E}}=\stackrel{\mathrm{E}}{r}_{\mathrm{c} / \mathrm{O}_{\mathrm{E}}}
$$

The notation used here is consistent with [14].

The angular velocity $\vec{\omega}_{\mathrm{AC} / \mathrm{E}}$ of $\mathrm{F}_{\mathrm{AC}}$ relative to $\mathrm{F}_{\mathrm{E}}$ is obtained from the rotation matrix $\vec{R}_{\mathrm{AC} / \mathrm{E}}$ by Poisson's equation

$$
\stackrel{\mathrm{AC}}{R}_{\mathrm{AC} / \mathrm{E}}=\vec{R}_{\mathrm{AC} / \mathrm{E}} \vec{\omega}_{\mathrm{AC} / \mathrm{E}}^{\times}
$$

We resolve $\vec{V}_{\mathrm{AC}}$ and $\vec{\omega}_{\mathrm{AC} / \mathrm{E}}$ using the notation

$$
\left.\left[\begin{array}{c}
U \\
V \\
W
\end{array}\right] \triangleq \vec{V}_{\mathrm{AC}}\right|_{\mathrm{AC}},\left.\quad\left[\begin{array}{c}
P \\
Q \\
R
\end{array}\right] \triangleq \vec{\omega}_{\mathrm{AC} / \mathrm{E}}\right|_{\mathrm{AC}} .
$$

Steady flight is defined as aircraft flight with constant $U, V, W, P, Q, R$.

\section{III.B. Trim Flight}

Trim flight is steady flight with additional constraints. Airspeed $V_{\mathrm{AC}}$ is defined as the magnitude of the velocity vector, that is,

$$
V_{\mathrm{AC}}=\left|\vec{V}_{\mathrm{AC}}\right|=\sqrt{U^{2}+V^{2}+W^{2}}
$$


Flight path angle $\gamma$ is defined as the angle between $\vec{V}_{\mathrm{AC}}$ and its projection onto the horizontal $\hat{\imath}_{\mathrm{E}} \hat{\jmath}_{\mathrm{E}}$ plane.

We define the orientation matrix of $\mathrm{F}_{\mathrm{AC}}$ relative to $\mathrm{F}_{\mathrm{E}}$ by

$$
\left.\mathcal{O}_{\mathrm{AC} / \mathrm{E}} \triangleq \vec{R}_{\mathrm{AC} / \mathrm{E}}\right|_{\mathrm{AC}} ^{\mathrm{T}}
$$

We then resolve the angular velocity in the Earth inertial frame by noting that

$$
\left.\vec{\omega}_{\mathrm{AC} / \mathrm{E}}\right|_{\mathrm{E}}=\left.\mathcal{O}_{\mathrm{AC} / \mathrm{E}}^{\mathrm{T}} \vec{\omega}_{\mathrm{AC} / \mathrm{E}}\right|_{\mathrm{AC}}=\left.\mathcal{O}_{\mathrm{E} / \mathrm{AC}} \vec{\omega}_{\mathrm{AC} / \mathrm{E}}\right|_{\mathrm{AC}}=\left.\mathcal{O}_{\mathrm{E} / \mathrm{E}^{\prime}} \mathcal{O}_{\mathrm{E}^{\prime} / \mathrm{E}^{\prime \prime}} \mathcal{O}_{\mathrm{E}^{\prime \prime} / \mathrm{AC}} \vec{\omega}_{\mathrm{AC} / \mathrm{E}}\right|_{\mathrm{AC}}
$$

and we define

$$
\left.\left[\begin{array}{c}
P_{\mathrm{E}} \\
Q_{\mathrm{E}} \\
R_{\mathrm{E}}
\end{array}\right] \triangleq \vec{\omega}_{\mathrm{AC} / \mathrm{E}}\right|_{\mathrm{E}} .
$$

Turn rate is $R_{\mathrm{E}}$.

Trim flight is defined as steady flight with constant flight path angle. Therefore, trim flight occurs if and only if airspeed, flight path angle, and turn rate are constant. During trim flight, forces on the aircraft are constant and balanced and control surface deflection and throttle settings are constant.

\section{Waypoint-Defined Trajectory Following}

In a flight safety management system, high level flight planning algorithms typically map emergency descent and landing trajectories by commanding a sequence of trim conditions [5]. In an emergency event, it is desirable for the aircraft to maintain this state until transition is deemed safe. In this section, we use RCAC to follow trim state transitions under minimal modeling information of the aircraft dynamics.

\section{IV.A. Achieving Trim Conditions}

Trim conditions can be classified into six types of flight conditions, namely, level straight-line flight, ascending straight-line flight, descending straight-line flight, level turning flight, rising-helical flight, and descending helical flight. These flight conditions can be constructed by commanding the following combinations of constant airspeed, flight path angle, and turn rate.

1. Level straight-line flight with positive airspeed, zero flight path angle, and zero turn-rate commands.

2. Rising straight-line flight with positive airspeed, positive flight path angle, and zero turn-rate commands. 
3. Descending straight-line flight with positive airspeed, negative flight path angle, and zero turn-rate commands.

4. Level turning flight with positive airspeed, zero flight path angle, and nonzero turn-rate commands.

5. Rising helical flight with positive airspeed, positive flight path angle, and nonzero turn-rate commands.

6. Descending helical flight with positive airspeed, negative flight path angle, and nonzero turn-rate commands.

\section{IV.B. Trim Condition Commands}

We now use RCAC to transition between trim conditions by following airspeed, flight path angle, and turn-rate commands. In all examples, the command is a trapezoidal command, with transitions occurring over $100 \mathrm{sec}$. For the controller tunings, $\eta_{0}$ is implemented as a vector in order to specify different weightings for different actuators. The first component of $\eta_{0}$ corresponds to the left throttle, the second component corresponds to the right throttle, the third component corresponds to the elevator, the fourth component corresponds to the ailerons, and the fifth component corresponds to the rudder.

A single tuning is used for all cases of trim transition commands, namely,

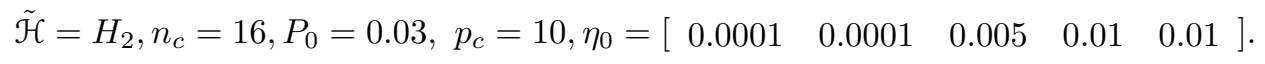

Example IV.1. Figure 1 shows the case where a trapezoidal command changes the airspeed by 10 $\mathrm{ft} / \mathrm{sec}$ while flight path angle and turn rate are kept constant. This command represents a transition from level, straight-line flight to another level, straight-line flight at an increased speed, and then back to the original trim state.

Example IV.2. Figure 2 shows the case where a trapezoidal command changes the flight path angle by 2 deg while airspeed and turn rate are kept constant. This command represents a transition from level, straight-line flight to a rising, straight-line flight, and then back to the original trim state.

Example IV.3. Figure 3 shows the case where a trapezoidal command changes the turn rate by 10 $\mathrm{deg} / \mathrm{sec}$ while airspeed and flight path angle are kept constant. This command represents a transition from level, straight-line flight to a level, turning flight, and then back to the original trim state.

Example IV.4. Figure 4 shows the case where a trapezoidal command increases the flight path angle by $2 \mathrm{deg}$ and the airspeed by $10 \mathrm{ft} / \mathrm{sec}$ while the turn rate is kept constant. This command represents a transition from level, straight-line flight to a rising, straight-line flight at an increased speed, and then back to the original trim state.

Example IV.5. Figure 5 shows the case where a trapezoidal command changes the flight path angle by $2 \mathrm{deg}$ and the turn rate by $2 \mathrm{deg} / \mathrm{sec}$ while the airspeed is kept constant. This command represents a transition from level, straight-line flight to a rising helical flight, and then back to the original trim state.

Example IV.6. Figure 6 shows the case where trapezoidal commands changes the airspeed by 10 $\mathrm{ft} / \mathrm{sec}$ and turn rate by $2 \mathrm{deg} / \mathrm{sec}$ while flight path angle is kept constant. This command represents a transition from level, straight-line flight to a level, turning flight at an increased speed, and then back to the original trim state.

Example IV.7. Figure 7 shows the case where trapezoidal commands changes the airspeed by 10 $\mathrm{ft} / \mathrm{sec}$, flight path angle by $2 \mathrm{deg}$, and turn rate by $2 \mathrm{deg} / \mathrm{sec}$. This command represents a transition from level, straight-line flight to a rising helical flight at an increased speed, and then back to the original trim state. 


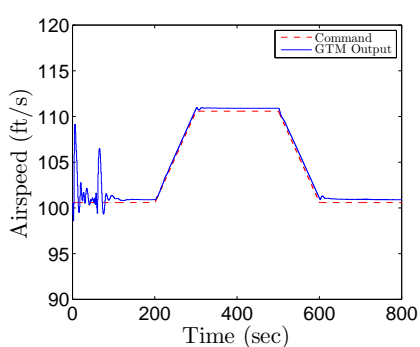

(a)

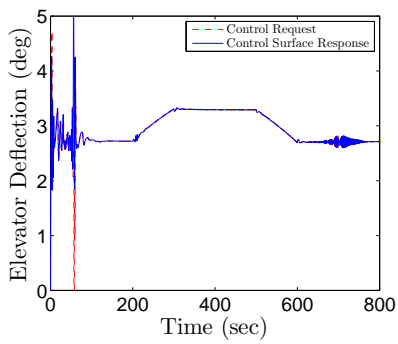

(d)

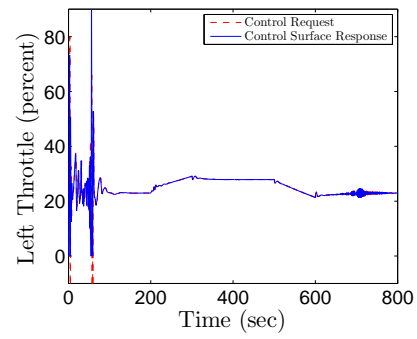

(g)

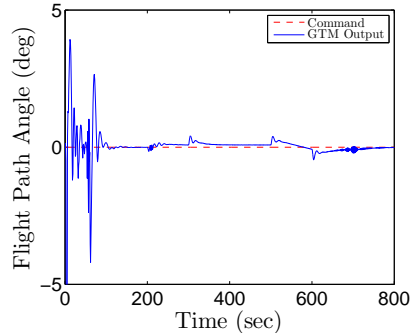

(b)

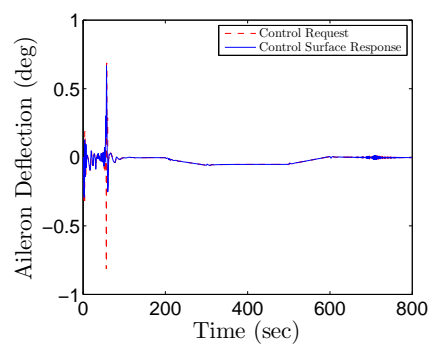

(e)

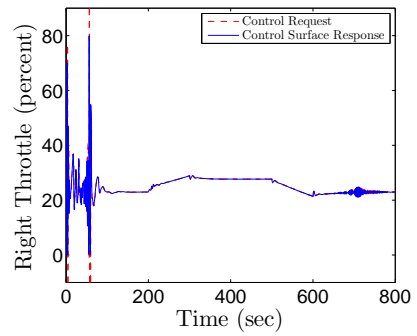

(h)

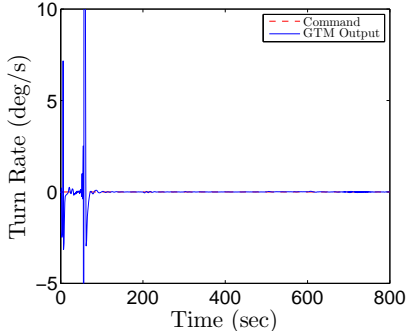

(c)

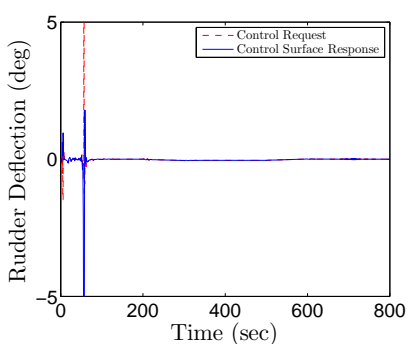

(f)

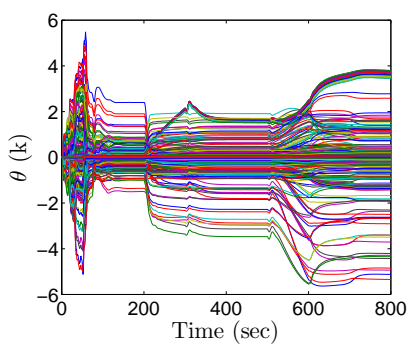

(i)

Figure 1. Example IV.1. Airspeed trapezoidal command, with zero flight-path-angle and zero turn-rate command. (a) airspeed, (b) flight path angle, (c) turn rate, (d) elevator deflection, (e) aileron deflection, (e) rudder deflection, (e) left throttle, (e) right throttle, and (f) controller gains. The controller is able to follow the command.

\section{Extended Trim Commands with Possibly Conflicting Parameters}

In this section we extend the trim command to include the angle of attack $\alpha$. Therefore, a trim command is now of the form

$$
\left[\begin{array}{llll}
V_{\mathrm{AC}} & \alpha & R_{\mathrm{E}} & \gamma
\end{array}\right]
$$

Since the static flight envelope can be specified in terms of either airspeed or angle of attack, commanded values of airspeed and angle of attack may be conflicting. Since the static flight envelope may be uncertain under off-nominal conditions, we command both of these parameters in order to ensure that both variables remain within a desirable range. Furthermore, since the static flight envelope is uncertain, we allow for the possibility that turn rate and flight path angle may also be conflicting. The goal is therefore to determine whether RCAC can achieve a feasible trim condition despite conflicting trim commands. We then adjust the weights associated with the trim parameters in order to influence the accuracy with which specific trim commands are followed.

In the retrospective cost $(22)$, we let $R(k)$ include a weight for each trim command parameter. We 


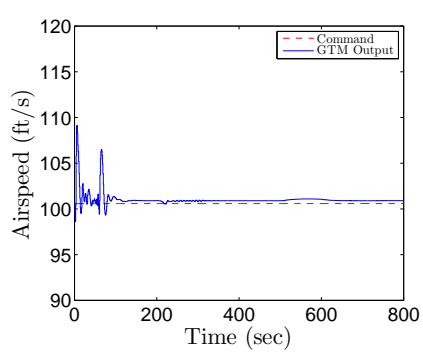

(a)

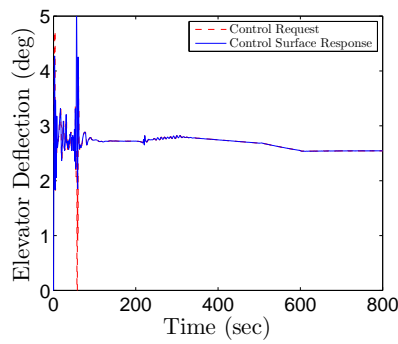

(d)

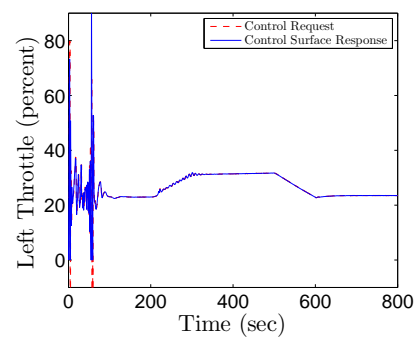

(g)

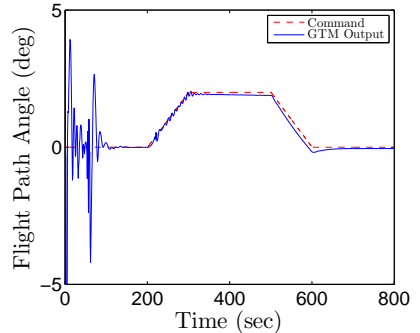

(b)

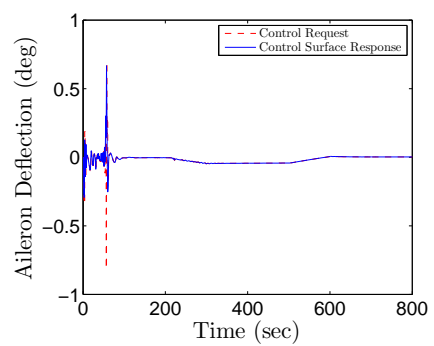

(e)

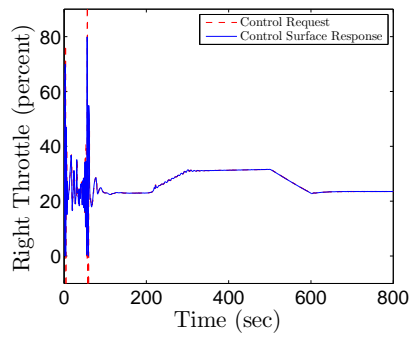

(h)

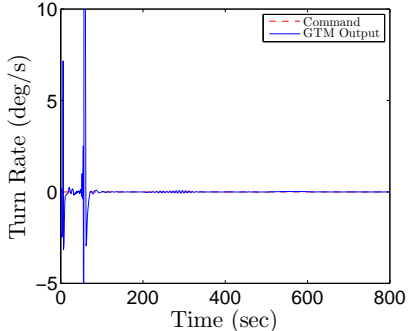

(c)

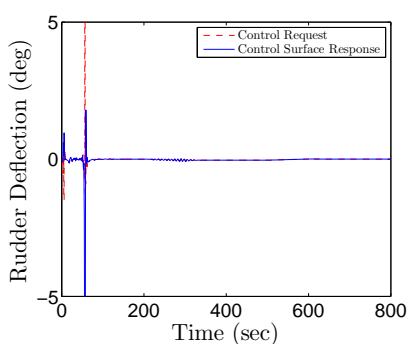

(f)

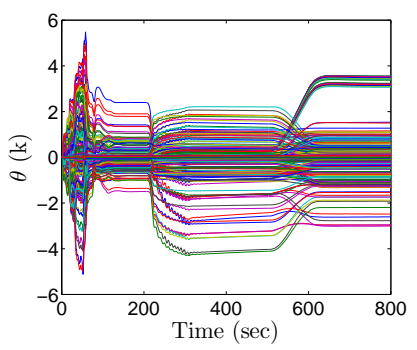

(i)

Figure 2. Example IV.2. Flight path angle trapezoidal command, while maintaining initial airspeed and zero turn-rate command. (a) airspeed, (b) flight path angle, (c) turn rate, (d) elevator deflection, (e) aileron deflection, (e) rudder deflection, (e) left throttle, (e) right throttle, and (f) controller gains. The controller is able to follow the command.

adjust $R(k)$ online to reflect the desired priority for each trim command parameter. For each example below,

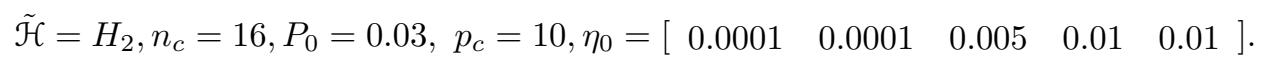

Example V.1. Extended Trim Command with Unknown Envelope. Let $R(k)$ be chosen as

$$
R(k)=\left\{\begin{array}{cccc}
\operatorname{diag}\left[\begin{array}{cccc}
1 & 0.0001 & 1 & 1
\end{array}\right], & T<600 \mathrm{sec} \\
\operatorname{diag}\left[\begin{array}{lllll}
0.01 & 400 & 1 & 1
\end{array}\right], & 600 \mathrm{sec} \leq T \leq 1000 \mathrm{sec} \\
\operatorname{diag}\left[\begin{array}{llll}
4 & 0.1 & 10 & 10
\end{array}\right], & T>1000 \mathrm{sec}
\end{array}\right.
$$

The entries in $R(k)$ represent the weights for $V_{\mathrm{AC}}, \alpha, R_{\mathrm{E}}$, and $\gamma$, respectively. Figure 8 shows the extended trim command following results.

Step 1: During 0-600 sec, we give a conflicting command at $200 \mathrm{sec}$, and the aircraft reaches a trim state. Note that only $V_{\mathrm{AC}}$ and $R_{\mathrm{E}}$ are followed accurately.

Step 2: During 600-1000 sec, we increase the weighting for the commanded $\alpha$ and force the aircraft to follow the commanded parameter $\alpha$.

Step 3: During 1000-1600 sec, we increase the flight path angle command and increase the weights 


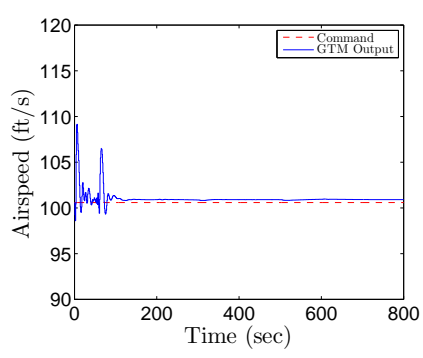

(a)

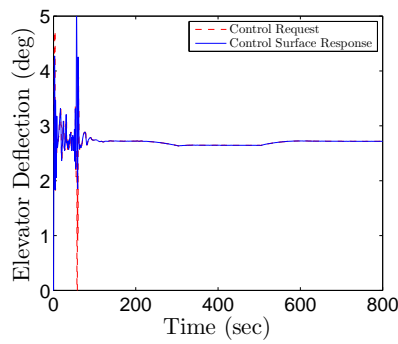

(d)

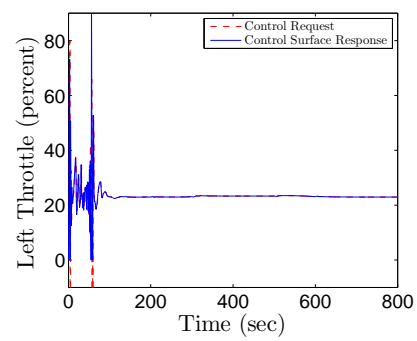

(g)

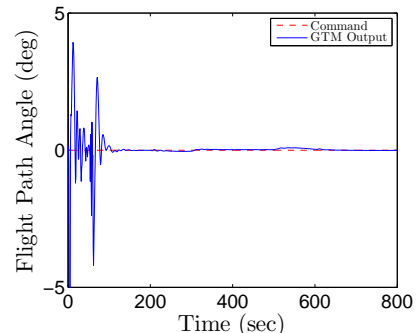

(b)

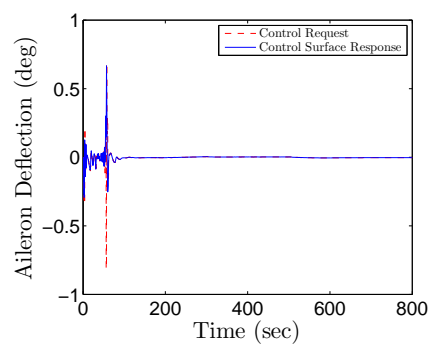

(e)

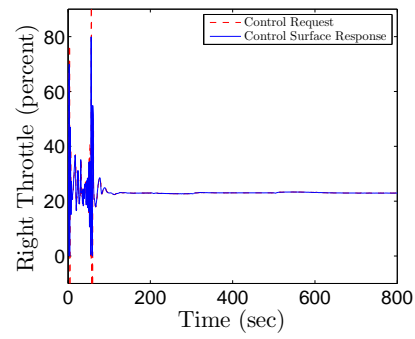

(h)

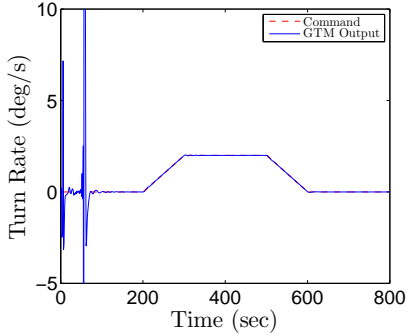

(c)

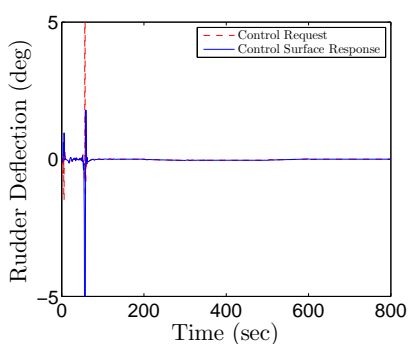

(f)

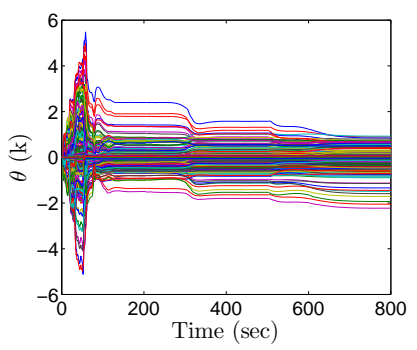

(i)

Figure 3. Example IV.3. Turn-rate trapezoidal command, while maintaining initial airspeed and zero flight-path-angle command. (a) airspeed, (b) flight path angle, (c) turn rate, (d) elevator deflection, (e) aileron deflection, (e) rudder deflection, (e) left throttle, (e) right throttle, and (f) controller gains. The controller is able to follow the command.

for $V_{\mathrm{AC}}, R_{\mathrm{E}}$, and $\gamma$ to force the aircraft to fly straight and upward. The aircraft reaches the trim state $\left[\begin{array}{llll}V_{\mathrm{AC}} & \alpha & R_{\mathrm{E}} & \gamma\end{array}\right]^{\prime}$.

Step 4: During 1600-2000 sec, we give [ $\left.\begin{array}{llll}V_{\mathrm{AC}} & \alpha & R_{\mathrm{E}} & \gamma\end{array}\right]^{\prime}$ as the extended trim command to demonstrate consistency.

Example V.2. Detecting Sensor Failure. We now consider an extended trim command in the presence of an unknown sensor failure. Figure 9 shows the case where an extended trim command is given and the airspeed sensor fails. $R(k)$ is chosen as

$$
R(k)=\left\{\begin{array}{rrrr}
\operatorname{diag}\left[\begin{array}{llll}
1 & 1 & 1 & 4
\end{array}\right], & T<250 \mathrm{sec} \\
\operatorname{diag}\left[\begin{array}{llll}
0 & 10 & 20 & 100
\end{array}\right], & 250 \mathrm{sec} \leq T
\end{array}\right.
$$

Step 1: 0-200 sec, RCAC keeps the aircraft in a trim state and all sensors operate correctly during this time interval.

Step 2: 200-250 sec, the pitot tube is faulty and provides an erroneous measurement bias of +30 ft/sec. The trim-parameter weightings are unchanged. The flight path angle decreases substantially. 


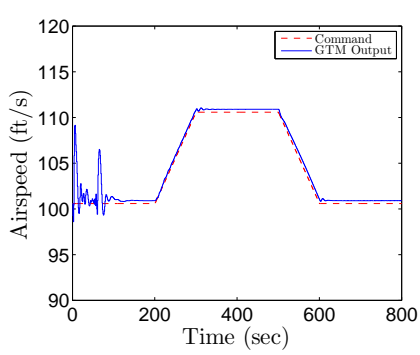

(a)

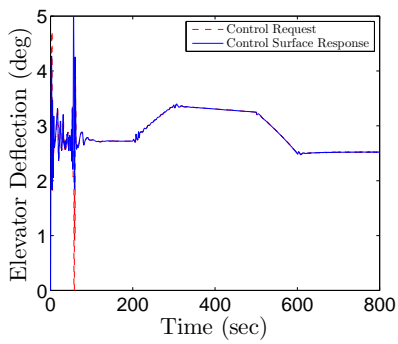

(d)

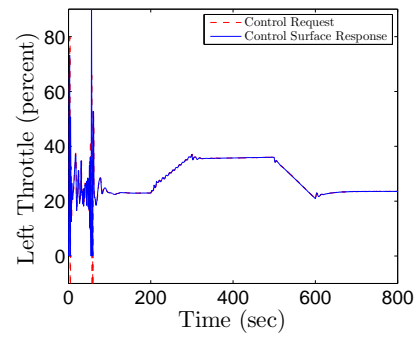

(g)

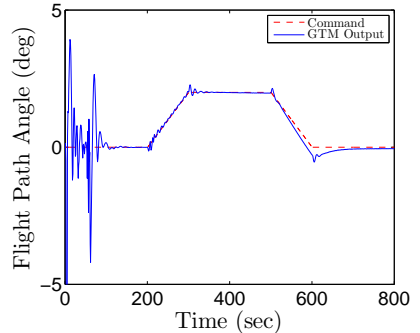

(b)

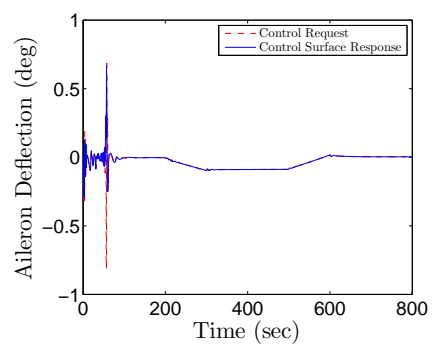

(e)

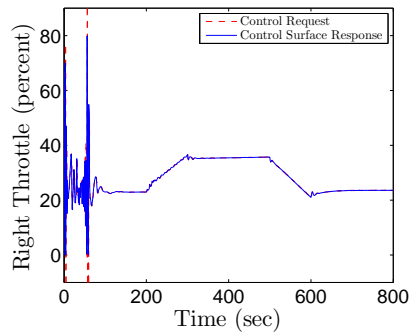

(h)

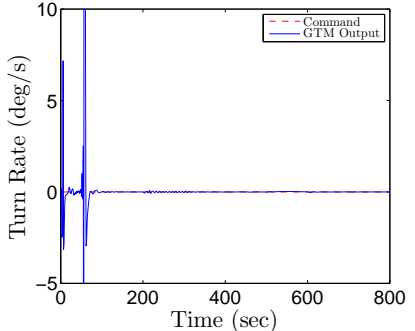

(c)

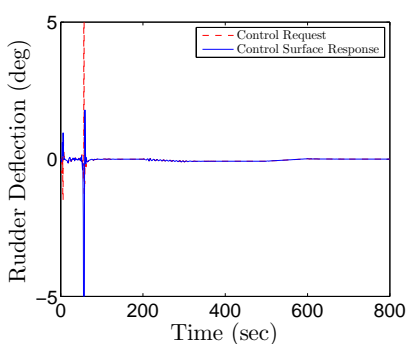

(f)

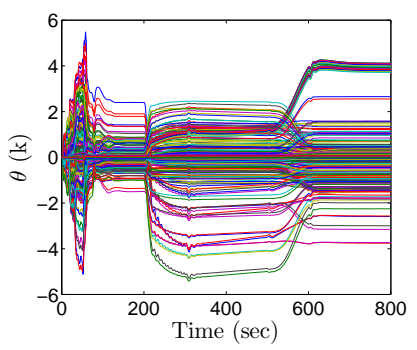

(i)

Figure 4. Example IV.4. Airspeed and flight-path-angle trapezoidal command, with zero turn rate-command. (a) airspeed, (b) flight path angle, (c) turn rate, (d) elevator deflection, (e) aileron deflection, (e) rudder deflection, (e) left throttle, (e) right throttle, and (f) controller gains. The controller is able to follow the commands.

Step 3: 250-800 sec, sensor failure is hypothesized based on altitude measurements. The weights for $\gamma, R_{\mathrm{E}}$, and $\alpha$ are increased, and the weight for $V_{\mathrm{AC}}$ is decreased to zero, effectively ignoring the pitot tube. Then the aircraft reaches the commanded trim state.

Example V.3. High Airspeed Test. We now consider an extended trim command in which the airspeed command is increased to an unreachable value. $R(k)$ is chosen as

$$
R(k)=\operatorname{diag}\left[\begin{array}{llll}
14 & 0.01 & 1 & 1
\end{array}\right] .
$$

Figure 10 shows the case where an unreachable airspeed command is given. From 100 sec, the airspeed is commanded to increase at the rate $1 \mathrm{ft} / \mathrm{sec}^{2}$. Figure 10 shows that the airspeed stops increasing at about $250 \mathrm{sec}$ and the aircraft converges to a trim state that matches the other three trim command parameters.

Example V.4. High Angle of Attack Test. We now consider an extended trim command in which commanded $\alpha$ is increased to an unreachable value. $R(k)$ is chosen as

$$
R(k)=\operatorname{diag}\left[\begin{array}{llll}
0.1 & 2000 & 1 & 1
\end{array}\right]
$$

Figure 11 shows the case where an unreachable angle of attack command is given. From 100 sec, commanded $\alpha$ increases at the rate $0.05 \mathrm{deg} / \mathrm{sec}^{2}$. Figure 11 shows that $\alpha$ stops increasing at about 300 sec and the aircraft converges to a new trim state. 


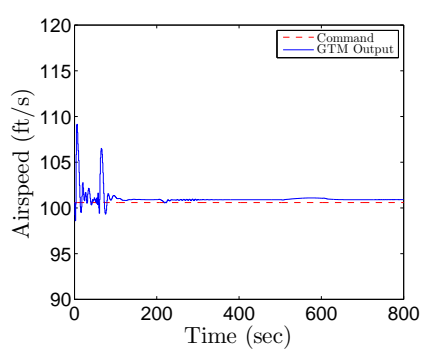

(a)

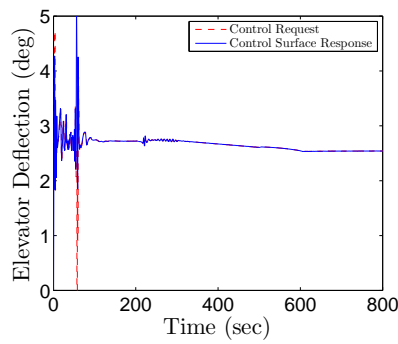

(d)

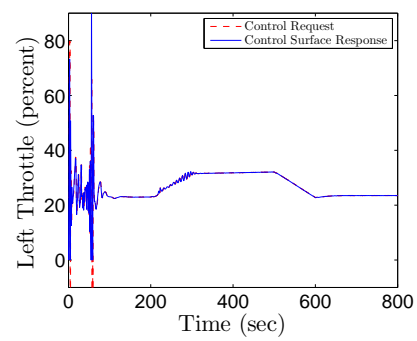

(g)

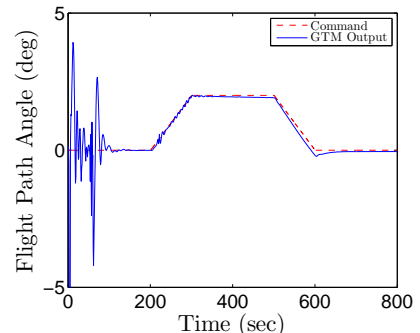

(b)

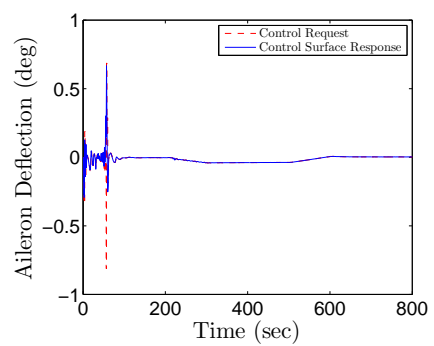

(e)

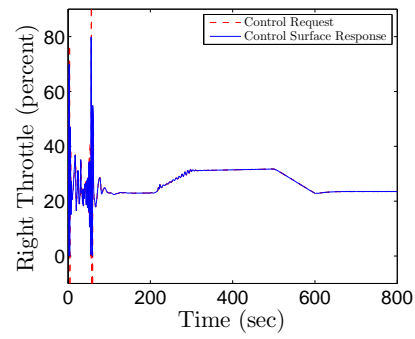

(h)

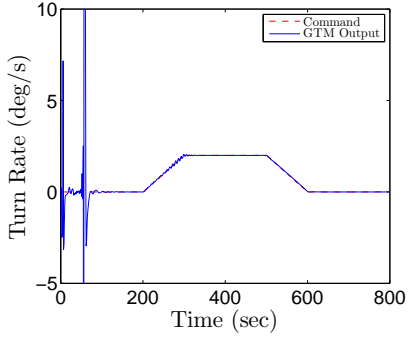

(c)

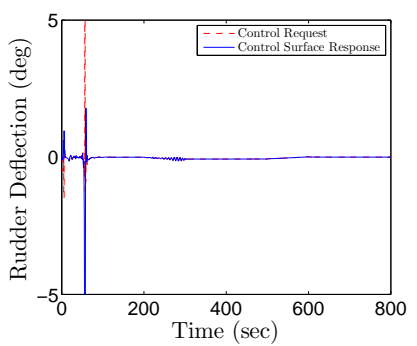

(f)

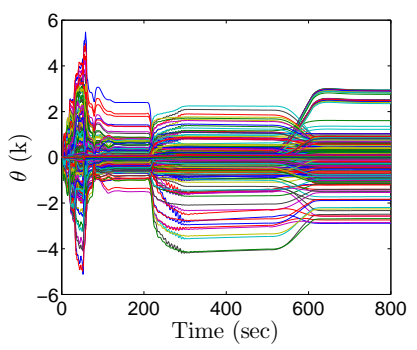

(i)

Figure 5. Example IV.5. Flight-path-angle and turn rate trapezoidal command, while maintaining initial airspeed command. (a) airspeed, (b) flight path angle, (c) turn rate, (d) elevator deflection, (e) aileron deflection, (e) rudder deflection, (e) left throttle, (e) right throttle, and (f) controller gains. The controller is able to follow the trim commands.

\section{Conclusions}

In this paper, we used RCAC for aircraft control in the context of an integrated flight safety management system. The controller commanded the aircraft to transition from trim state to trim state, including level straight-line flight, rising straight-line flight, level turning flight, and rising helical flight. We also demonstrated robustness in following conflicting trim commands for the case of an unknown envelope under system faults. 


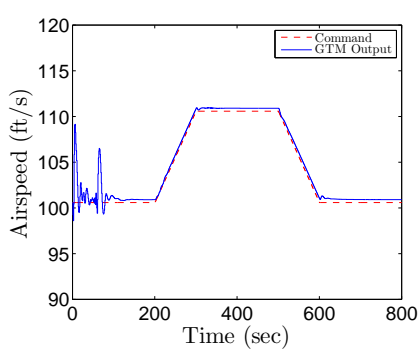

(a)

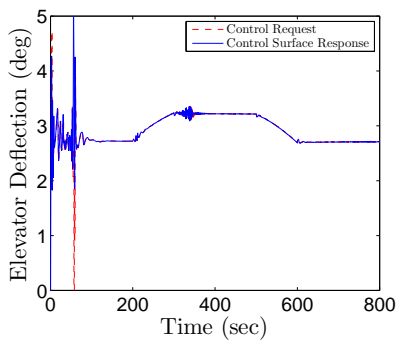

(d)

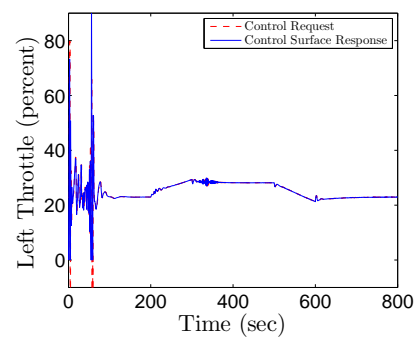

(g)

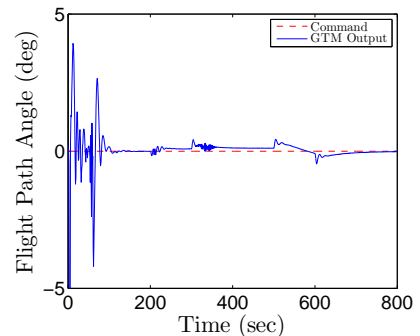

(b)

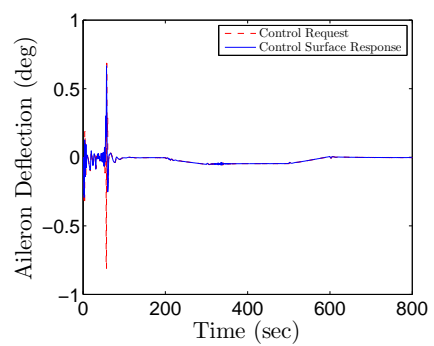

(e)

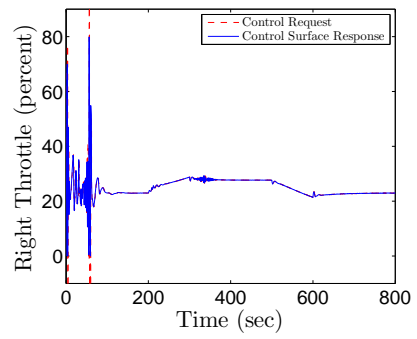

(h)

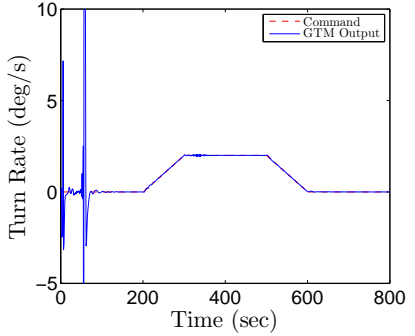

(c)

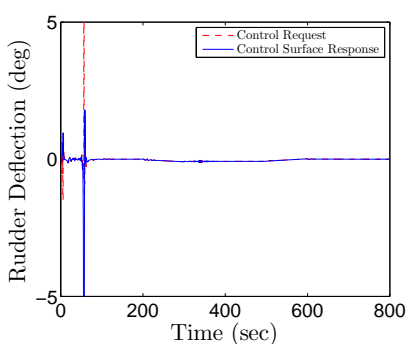

(f)

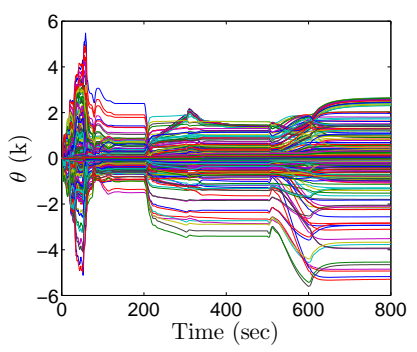

(i)

Figure 6. Example IV.6. Airspeed and turn rate trapezoidal command, with zero flight-path-angle command. (a) airspeed, (b) flight path angle, (c) turn rate, (d) elevator deflection, (e) aileron deflection, (e) rudder deflection, (e) left throttle, (e) right throttle, and (f) controller gains. The controller is able to follow the trim command.

\section{Acknowledgment}

This work was supported in part by the National Aeronautics and Space Administration under Cooperative Agreement NNX12AM54A.

\section{References}

${ }^{1}$ Dubins, L. E., "On curves of minimal length with a constraint on average curvature, and with prescribed initial and terminal positions and tangents," American Journal of Mathematics, Vol. 79, 1957, pp. 497-516.

${ }^{2}$ Shkel, A. M. and Lumelsky, V. J., "Classification of the Dubins set," Robotics and Autonomous Systems, 2001, pp. 179-202.

${ }^{3} \mathrm{Ma}$, X. and Castanon, D. A., "Receding Horizon Planning for Dubins Traveling Salesman Problems," San Diego, CA, December 2006, pp. 5453-5458.

${ }^{4}$ Techy, L. and Woolsey, C. A., "Minimum-Time Path Planning for Unmanned Aerial Vehicles in Steady Uniform Winds," AIAA J. Guid. Contr. Dyn., Vol. 32, 2009, pp. 1736-1746.

${ }^{5}$ Tang, Y., Atkins, E., and Sanner, R., "Emergency flight planning for a generalized transport aircraft with left wing damage," Proc. Guidance, Navigation, and Control Conference, 2007.

${ }^{6}$ Venugopal, R. and Bernstein, D. S., "Adaptive Disturbance Rejection Using ARMARKOV System Representations," IEEE Trans. Contr. Sys. Tech., Vol. 8, 2000, pp. 257-269.

${ }^{7}$ Hoagg, J. B., Santillo, M. A., and Bernstein, D. S., "Discrete-Time Adaptive Command Following and Disturbance 


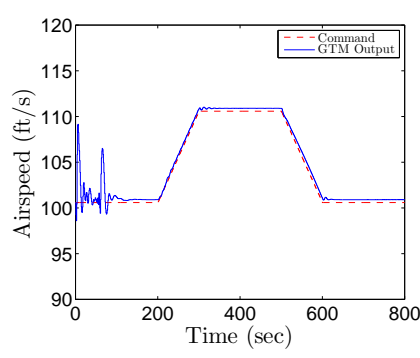

(a)

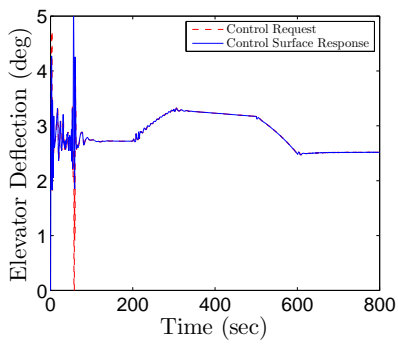

(d)

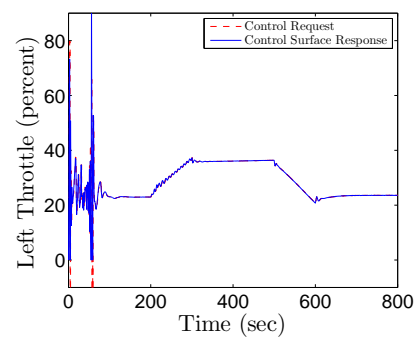

(g)

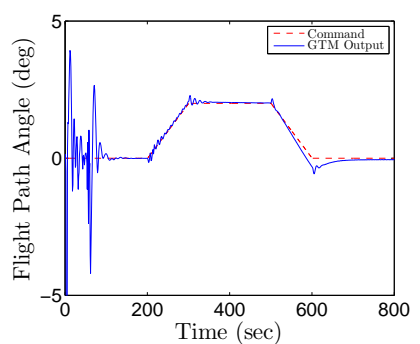

(b)

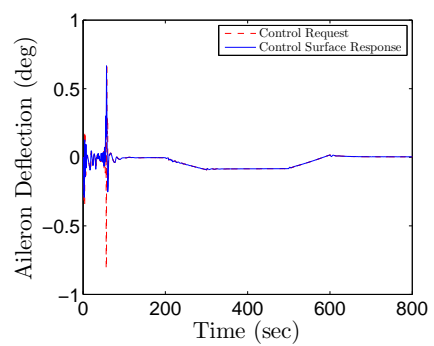

(e)

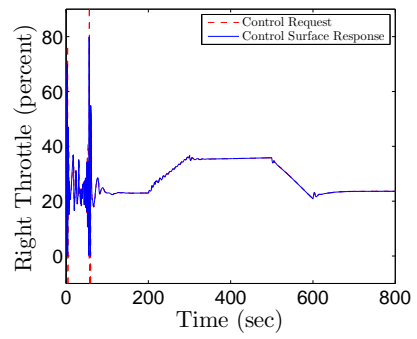

(h)

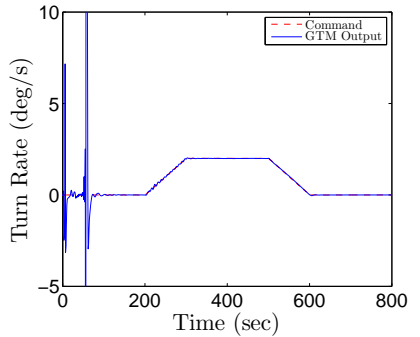

(c)

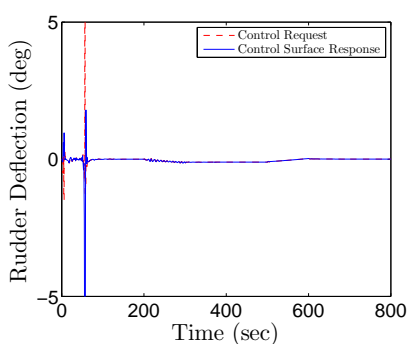

(f)

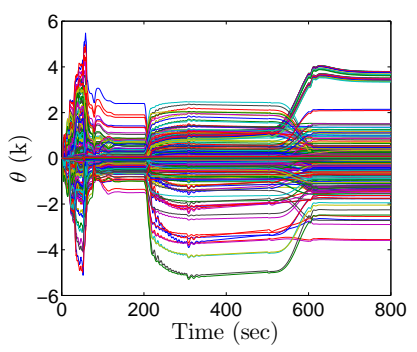

(i)

Figure 7. Example IV.7. Airspeed, flight path angle, and turn rate trapezoidal command. (a) airspeed, (b) flight path angle, (c) turn rate, (d) elevator deflection, (e) aileron deflection, (e) rudder deflection, (e) left throttle, (e) right throttle, and (f) controller gains. The controller is able to follow the trim command.

Rejection for Minimum Phase Systems with Unknown Exogenous Dynamics," IEEE Trans. Autom. Contr., Vol. 53, 2008, pp. $912-928$.

${ }^{8}$ Santillo, M. A. and Bernstein, D. S., "Adaptive Control Based on Retrospective Cost Optimization," AIAA J. Guid. Contr. Dyn., Vol. 33, 2010, pp. 289-304.

${ }^{9}$ Hoagg, J. B. and Bernstein, D., "Retrospective Cost Model Reference Adaptive Control for Nonminimum-Phase Systems," AIAA J. Guid. Contr. Dyn., Vol. 35, 2012, pp. 1767-1786.

${ }^{10}$ D'Amato, A. M., Sumer, E. D., and Bernstein, D. S., "Frequency-Domain Stability Analysis of Retrospective-Cost Adaptive Control for Systems with Unknown Nonminimum-Phase Zeros," Proc. IEEE Conf. Dec. Contr., Orlando, FL, December 2011, pp. 1098-1103.

${ }^{11}$ D'Amato, A. M., Sumer, E. D., Mitchell, K. S., Morozov, A. V., Hoagg, J. B., and Bernstein, D. S., "Adaptive Output Feedback Control of the NASA GTM Model with Unknown Nonminimum-Phase Zeros," Proc. AIAA Guid. Nav. Contr. Conf., Portland, OR, August 2011, AIAA-2011-6204.

${ }^{12}$ Bailey, R. and Jordan, T., "NASA Langley's AirSTAR Testbed: A Subscale Flight Test Capability for Flight Dynamics and Control System Experiments," Proc. AIAA Guid. Nav. Contr. Conf., Honolulu, HI, August 2008, AIAA-2008-6660.

${ }^{13}$ Murch, A., "A Flight Control System Architecture for the NASA AirSTAR Flight Test Infrastructure," Honolulu, HI, August 2008, AIAA-2008-6990.

${ }^{14}$ Yan, J., Hoagg, J. B., Hindman, R. E., and Bernstein, D. S., "Longitudinal Aircraft Dynamics and the Instantaneous Acceleration Center of Rotation: The Case of the Vanishing Zeros," IEEE Contr. Sys. Mag., Vol. 30, 2011, pp. 68-92. 

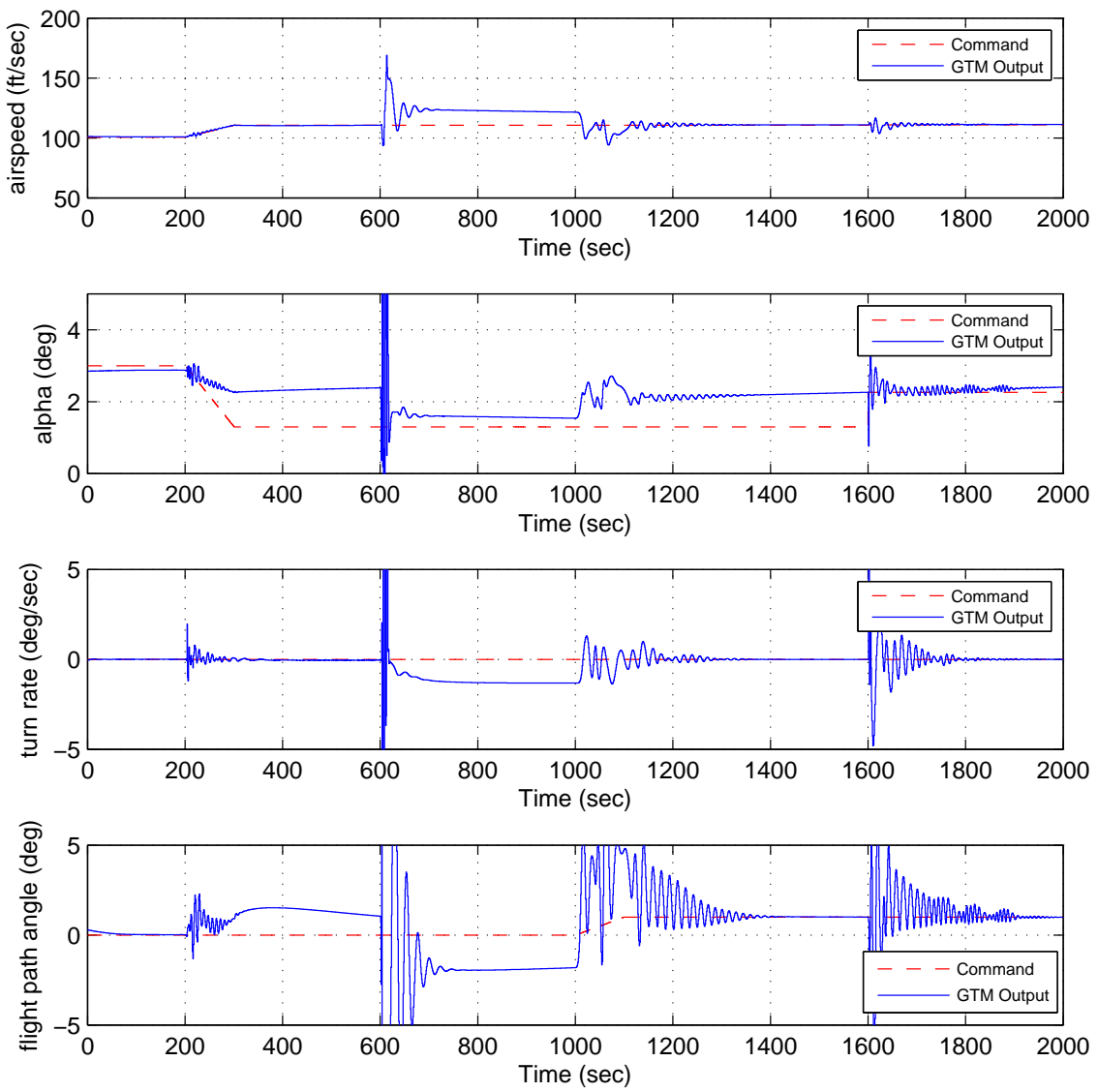

Figure 8. Example V.1. Extended trim command following with unknown envelope. The controller is able to follow the trim command as $R(k)$ is adjusted. 

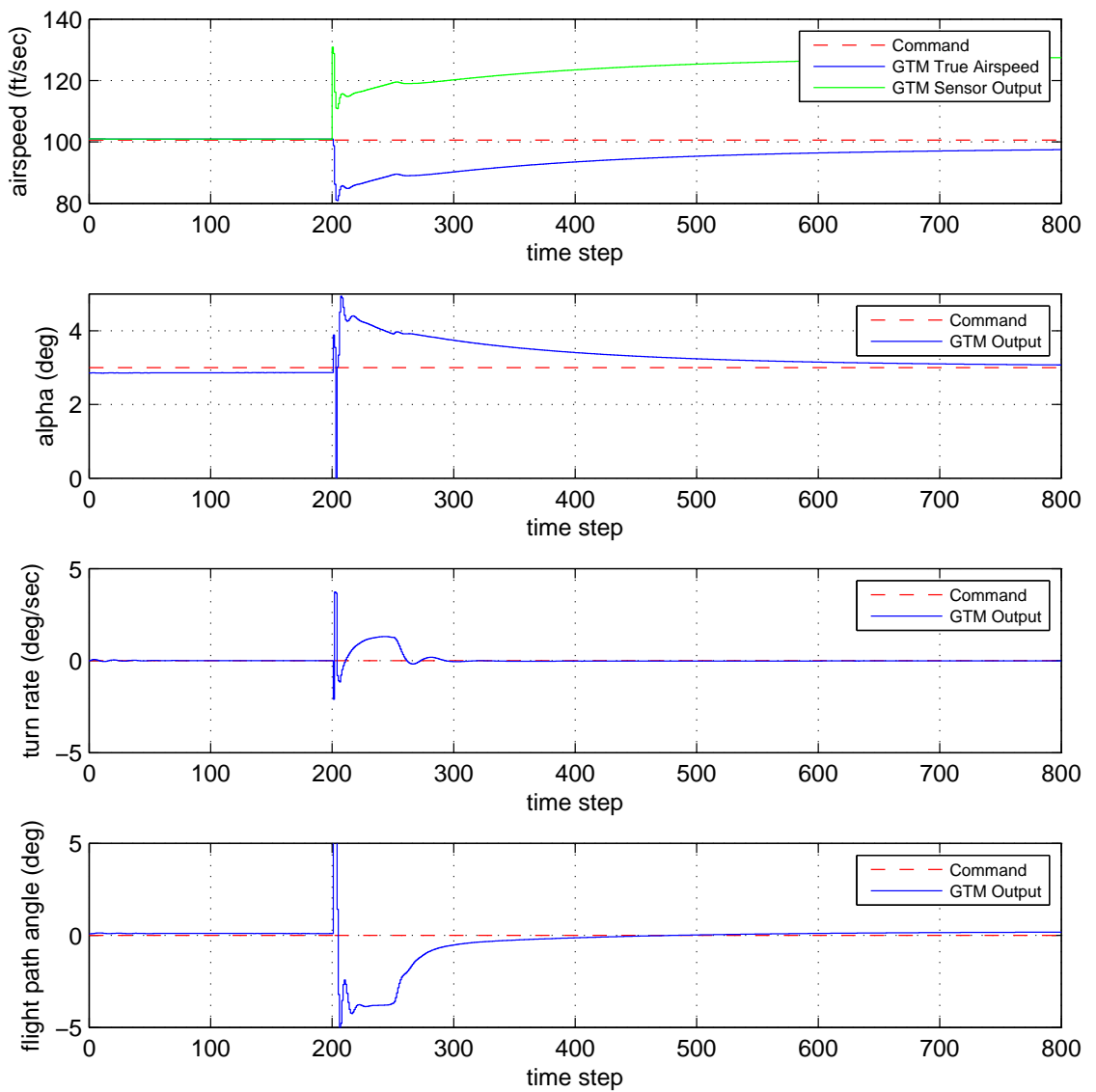

Figure 9. Example V.2. Extended trim command following with airspeed sensor failure. When airspeed sensor failure is detected, the controller is able to change guidance mode quickly by adjusting the weightings for command parameters to avoid the effect of sensor failure. 

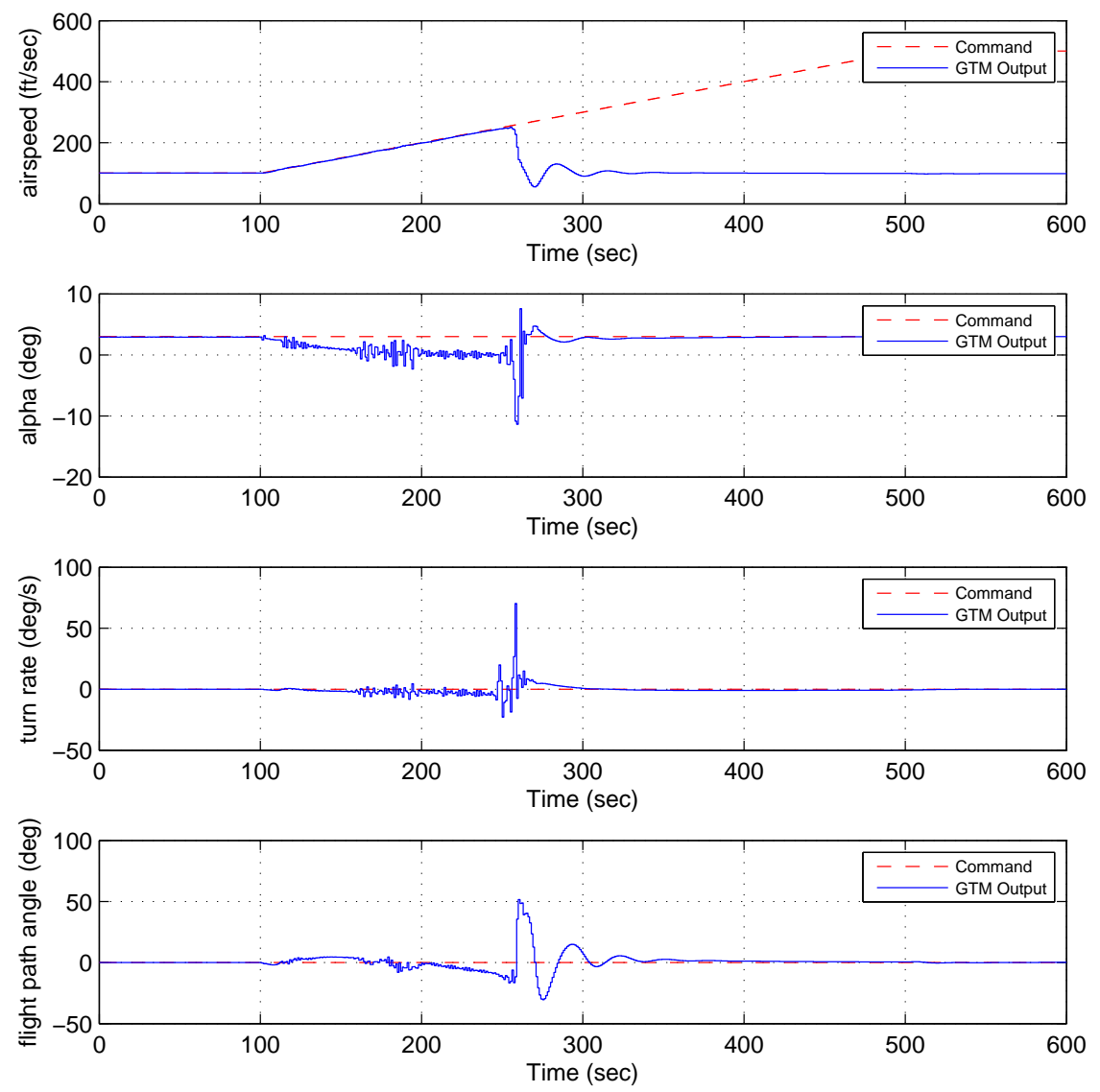

Figure 10. Example V.3. High airspeed test. When the airspeed reaches a value that makes the other three command parameters begin to oscillate, the aircraft stops following the airspeed command and converges to a trim state that matches the other three trim command parameters. 

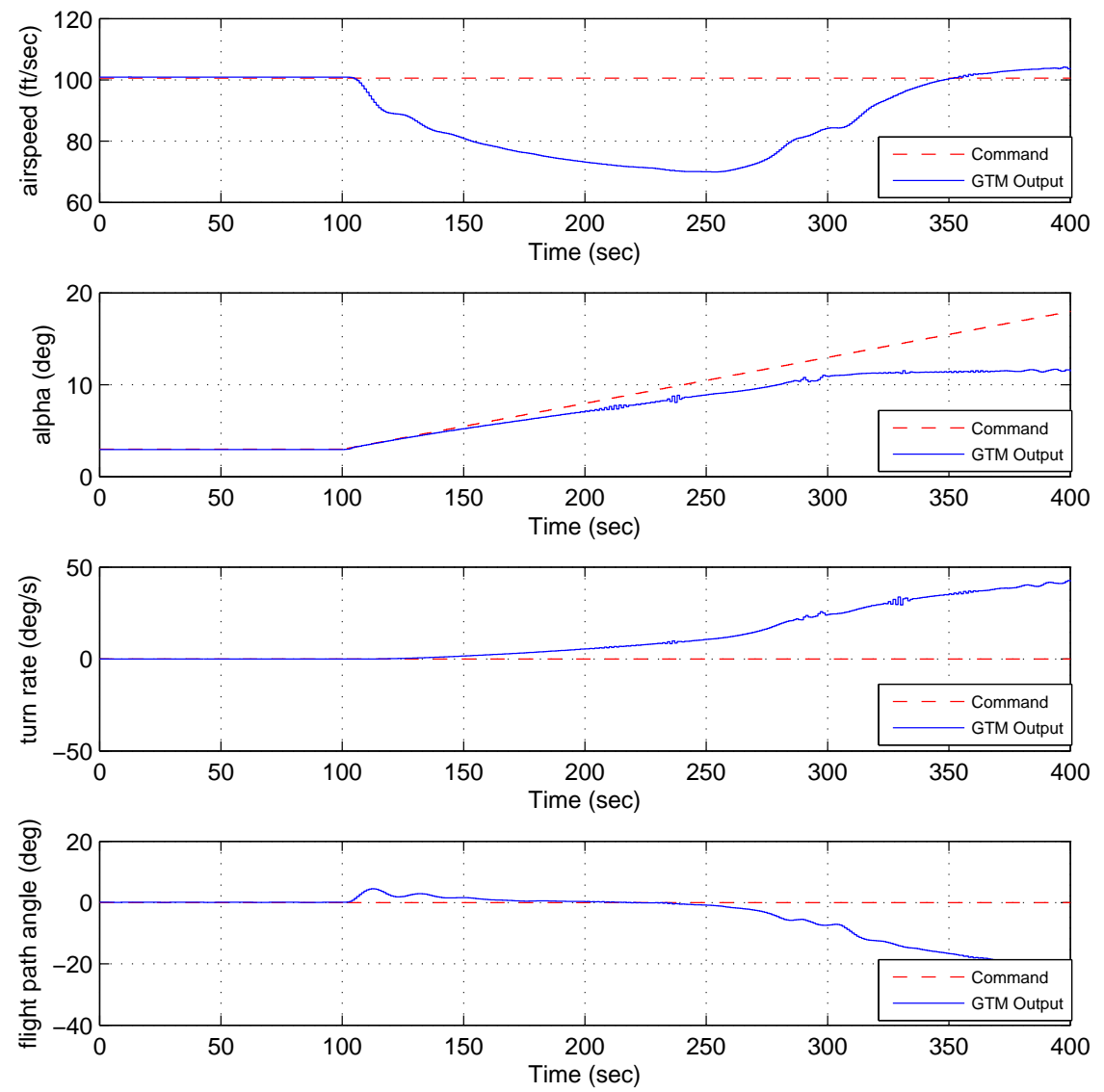

Figure 11. Example V.4. High angle of attack test. When $\alpha$ reaches a certain value, the aircraft stops following the commanded $\alpha$ and converges to a new trim state. 